\title{
In vitro cytotoxicity of combinations of dichloroacetate with anticancer platinum compounds
}

This article was published in the following Dove Press journal:

Clinical Pharmacology: Advances and Applications

14 September 2010

Number of times this article has been viewed

\author{
Ulrike Olszewski' \\ Thomas Tuxen Poulsen ${ }^{2}$ \\ Ernst Ulsperger' \\ Hans Skovgard Poulsen ${ }^{2}$ \\ Klaus Geissler' \\ Gerhard Hamilton' \\ 'Ludwig Boltzmann Cluster of \\ Translational Oncology, Ludwig \\ Boltzmann Society, Vienna, Austria; \\ ${ }^{2}$ Department of Radiation Biology, \\ Finsen Center, Copenhagen University \\ Hospital, Copenhagen, Denmark
}

Purpose: Dichloroacetate (DCA) inhibits pyruvate dehydrogenase kinase (PDK), and thus promotes glucose oxidation over glycolysis and induces apoptotic death of tumor cells. The present study investigated the potential of DCA to increase the antitumor effects of platinum-based compounds against a panel of permanent cell lines, including small cell lung cancer (SCLC), ovarian cancer, and Ewing's sarcoma in vitro.

Methods: DCA at a concentration of $10 \mathrm{mM}$ was combined with cisplatin, carboplatin, satraplatin, the satraplatin metabolite JM118, oxaliplatin, oxoplatin, and picoplatin, and the cytotoxic activity was evaluated in proliferation tests employing a panel of different cell lines. Additionally, cells were pretreated with DCA and then exposed to the platinum drugs and etoposide, or incubated with cisplatin or etoposide followed by application of DCA, respectively.

Results: DCA $10 \mathrm{mM}$ significantly increased the cytotoxicity of the platinum-based drugs carboplatin, satraplatin, JM118, and oxoplatin, but not cisplatin, picoplatin, and oxaliplatin in vitro. Preincubation of cell lines with DCA $10 \mathrm{mM}$ for three days reduced the antiproliferative activity of platinum-based agents in sequential application, but exposure of cells pretreated with cisplatin or etoposide to DCA resulted in minor sensitization. The inhibitory effect of DCA showed no correlation with sensitization to the platinum compounds.

Conclusion: DCA alone in a concentration that shows low antiproliferative activity is capable of increasing the cytotoxicity of selected platinum compounds upon coincubation, and such combinations may be interesting for clinical application in tumors like SCLC, Ewing's sarcoma, and ovarian cancer refractory to cisplatin chemotherapy as standard care. The mechanism of this synergistic effect of DCA in combination with specific platinum species remains to be investigated.

Keywords: dichloroacetate, platinum, cytotoxicity, glycolysis, apoptosis

\section{Introduction}

Unlike normal, untransformed cells, most cancer cells rely on aerobic glycolysis to gain energy, a phenomenon termed the "Warburg effect". ${ }^{1}$ Since this is an inefficient way to generate adenosine triphosphate, this metabolic switch is supposed to confer other important advantages on tumor cells, such as capability to modulate intracellular/extracellular $\mathrm{pH}$, promote anabolic metabolism and, especially, the glycolytic phenotype in cancer, which suppresses mitochondrial function and thus increases resistance to apoptotic cell death. ${ }^{2}$ This direction of glucose utilization may be diverted by the generic drug dichloroacetate (DCA), an orally active small molecule that, by inhibiting pyruvate dehydrogenase kinase (PDK), increases the flux of pyruvate into the mitochondria and thus promotes glucose oxidation over glycolysis. ${ }^{3}$ Inhibition of in vitro and in vivo tumor growth by DCA is attributed to depressed mitochondrial function and increased apoptosis.
Correspondence: Gerhard Hamilton Ludwig Boltzmann Cluster of Translational Oncology, c/o Balderichgasse 26/13, A-I I 70 Vienna, Austria Tel $+43 \quad 1404006627$

Fax +43 I40 4006627

Email gerhard.hamilton@toc.lbg.ac.at 
DCA treatment promotes apoptosis in lung and breast cancer as well as glioblastoma cell lines by a shift in metabolism from aerobic glycolysis to glucose oxidation, coupled with tumor-specific decreases of membrane potential and activation of potassium channels in mitochondria. ${ }^{4}$ DCA-sensitive endometrial cancer cell lines treated with DCA 5-10 mM showed increased early and late apoptosis, decreased mitochondrial membrane potential and lower survivin transcript levels, consistent with a mitochondria-regulated mechanism. ${ }^{5}$ Treatment of representative colorectal cancer cell lines with DCA $20 \mathrm{mM}$ caused significant decreases in cancer cell proliferation associated with cell-cycle arrest in G2 phase and apoptosis, in contrast to noncancerous cell lines. ${ }^{6}$ The growth of several breast cancer cell lines was found to be inhibited by DCA in vitro. ${ }^{7}$ Furthermore, treatment of metastatic 13762 MAT rat mammary adenocarcinoma cells with DCA in vivo revealed that reversal of the glycolytic phenotype was correlated with inhibition of proliferation without affecting cell viability. These data demonstrate antiproliferative and proapoptotic properties for DCA, as well as antitumor activity against highly metastatic disease in vivo.

Although this drug seems generally safe, a clinical trial based on application of DCA $25 \mathrm{mg} / \mathrm{kg} /$ day was discontinued early because patients with MELAS (Mitochondrial Encephalopathy, Lactic Acidosis, and Stroke-like episodes) under treatment developed peripheral neuropathy. ${ }^{8}$ DCA is a major byproduct of water disinfection by chlorination, and several studies have demonstrated hepatocarcinogenesis in mice after intake through drinking water. ${ }^{9}$

An important mechanism of resistance acting downstream of the uptake of cisplatin and DNA adduct formation is decreased induction of apoptosis and, therefore, DCA-induced sensitization of cancer cells to this form of cell death is expected to overcome resistance partially. ${ }^{10}$ In an attempt to increase the cytotoxicity of cisplatin, a derivative was synthesized by coordination of two DCA molecules to the axial positions of this drug, yielding the platinum (IV) compound mitaplatin. ${ }^{11}$ This drug is cleaved in the cytoplasm, and the separated agents, cisplatin and DCA, attack nuclear DNA and mitochondria, respectively. The cytotoxicity of mitaplatin in a variety of cancer cell lines equals or exceeds that of all known platinum (IV) compounds and is comparable with that of cisplatin, partially overcoming drug resistance. However, with the use of mitaplatin, the platinum:DCA stoichiometry is invariably $1: 2$, with simultaneous application and DCA concentrations in tumor tissue in the subnanomolar range. ${ }^{12}$ Data on the intracellular concentration of DCA are lacking, but it is known that inhibition of the four isoforms of PDK as observed in treated cells requires the presence of DCA in the $0.2-8 \mathrm{mM} \mathrm{K}_{\mathrm{i}}$ range. ${ }^{13}$ Moreover, several problems still remain to be addressed regarding the most suitable platinum agent for combination with DCA, as well as the optimal concentration and sequence of DCA application. The effects of DCA on the mitochondrial membrane potential, cell viability, and induction of apoptosis were evaluated in pediatric tumor cell lines and in the nonmalignant cell line, HEK293. ${ }^{14}$ Concentrations of DCA $\leq 10 \mathrm{mM}$ only moderately inhibited growth of the cell lines, but the toxicity of cisplatin was abrogated in most cell lines, without effects on cisplatin stability and uptake.

We found in our laboratory that DCA acts synergistically with several platinum drugs, but not with cisplatin, in a chemoresistant human pulmonary carcinoid cell line. ${ }^{15}$ Therefore, this research was extended, and included combinations of DCA with a range of newer platinum drugs and different modes of sequential application in a larger panel of cancer cell lines and in HEK293.

\section{Materials and methods Chemicals and cell lines}

Unless otherwise noted, all chemicals and solutions were obtained from Sigma-Aldrich (St. Louis, MO). Satraplatin [bisacetatoammine dichloro cyclohexylamine platinum (IV)], JM118 [cis-ammine dichloro cyclohexylamine platinum(II)], oxoplatin [cis,cis,trans-diammine dichloro dihydroxo platinum (IV)], and picoplatin ([cis-ammine dichloro 2-methylpyridine platinum (II)] were custom synthesized. A $1 \mathrm{M}$ stock solution of sodium DCA was prepared by dissolution in phosphatebuffered saline. Cell lines were purchased from the American Tissue Culture Collection (ATCC, Manassas, VA) or the European Collection of Cell Cultures (ECACC, Porton Down, Salisbury, UK), respectively, and the Ewing's sarcoma and HEK293 cells were obtained from Dr T Thalhammer (Institute of Pathophysiology, University School of Medicine, Vienna, Austria). Cells were cultured in RPMI-1640 medium supplemented with $10 \%$ fetal bovine serum (Seromed, Berlin, Germany), $4 \mathrm{mM}$ glutamine, and antibiotics. HEK293, UMC-11, ES-2, and COLO 205 cells were subcultured by trypsinization (2.5\% trypsin/ethylenediaminetetraacetic acid solution; Boehringer Mannheim, Germany), and TC71 and TC252 cells were detached using $\mathrm{Ca}^{2+} \mathrm{Mg}^{2+}$-free phosphatebuffered saline.

\section{Cell proliferation assay}

Cells were harvested, counted, and distributed into the wells of flat-bottomed 96-well microtiter plates at a density of $1 \times 10^{4}$ cells/well in $100 \mu \mathrm{L}$ medium; $100 \mu \mathrm{L}$ of appropriate 
dilutions of test compounds were added to each well and plates incubated under tissue culture conditions for four days. Stock solutions of the compounds were prepared in either $70 \%$ ethanol or dimethyl sulfoxide, respectively, and diluted more than 100-fold for use in chemosensitivity assays. Solvent control wells were included in all tests. Dose-response curves were obtained by assessment of cell growth at twofold drug dilutions in triplicate and used for calculation of the $\mathrm{IC}_{50}$ half maximal inhibitory concentration values. Cell proliferation was quantified using a modified tetrazolium dye assay (MTT; EZ4U, Biomedica, Vienna, Austria) by measurement of the absorbance of the resulting dye at a wavelength of $450 \mathrm{~nm}$ (medium/solvent control set to $100 \%$ proliferation).

\section{Statistical analysis}

$\mathrm{IC}_{50}$ values and corresponding ratios were calculated from dose-response curves of the chemosensitivity assays performed in triplicate. A value of $P<0.05$ was regarded as statistically significant in $t$-tests. All calculations were performed using Winks statistical software (Texasoft, Duncanville, TX).

\section{Results}

According to the literature and our conclusions from initial titration experiments, concentrations of DCA below and exceeding $10 \mathrm{mM}$ resulted in insufficient activity or antagonistic growth inhibition when combined with chemotherapeutics, respectively, and, therefore, a DCA concentration of $10 \mathrm{mM}$ was used in all further experiments. The results of experiments investigating the cytotoxicity of a large panel of platinum drugs and etoposide in combination with $10 \mathrm{mM}$ DCA against DMS153 small cell lung cancer (SCLC) cells are shown in Figure 1. DCA alone inhibited the growth of DMS153 cells by $22.5 \% \pm 6.1 \%$. Inclusion of DCA had minor effects on the activity of cisplatin [cis-diammine dichloroplatinum(II)], oxaliplatin [1R,2R-diaminocyclohexane oxalato platinum (II)], and picoplatin, whereas the $\mathrm{IC}_{50}$ values of carboplatin [cis-diammine (1,1-cyclobutane dicarboxylato) platinum(II)], satraplatin, the satraplatin metabolite JM118, and oxoplatin were significantly lowered, indicating considerable sensitization of the cells to selected platinum complexes. In addition, etoposide showed increased cytotoxicity in the presence of DCA. In a similar

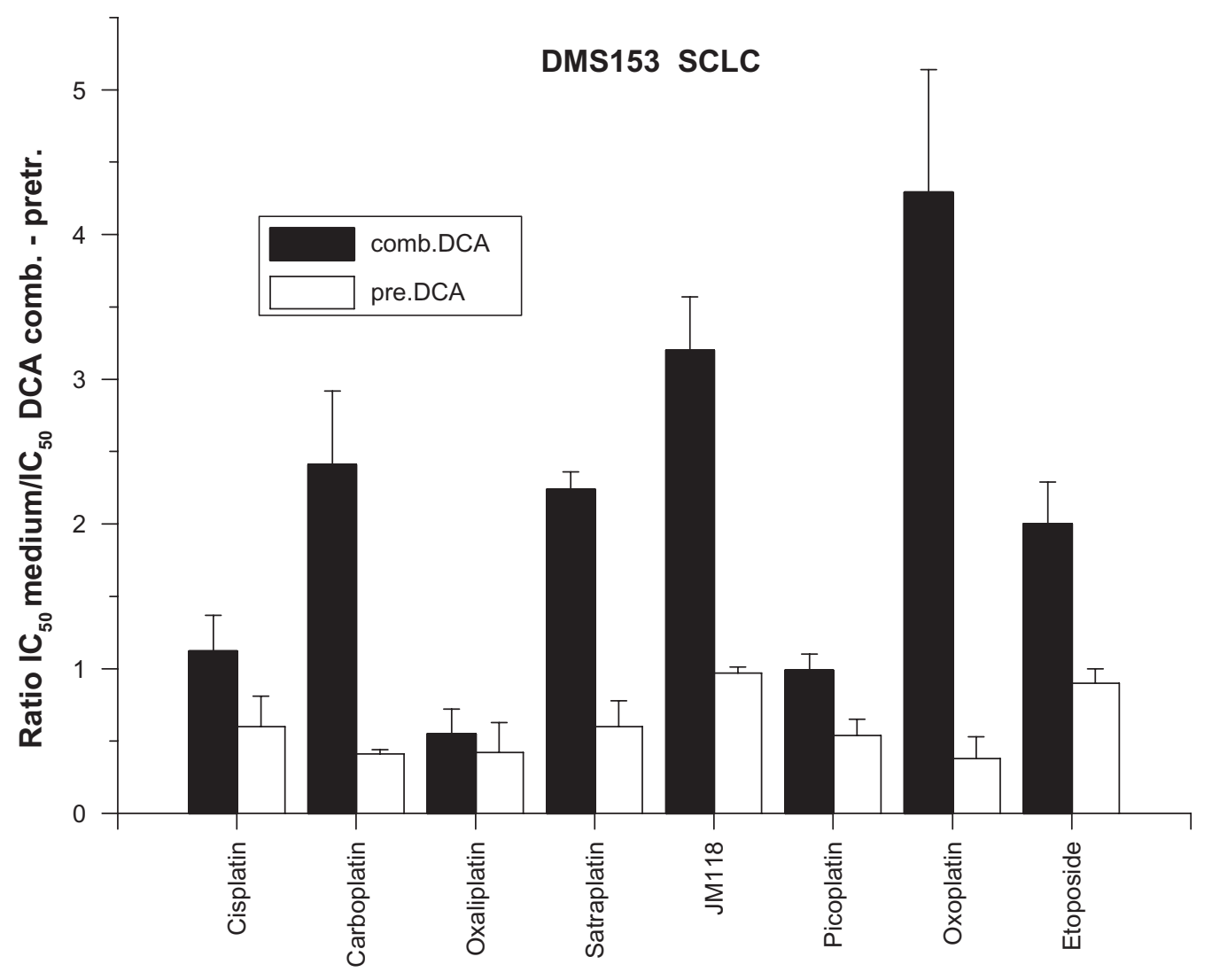

Platinum compound

Figure I Sensitization of DMS I53 SCLC cells by either combined treatment with DCA $10 \mathrm{mM}$ and platinum drugs or following pretreatment of cells with DCA 10 mM (mean \pm standard deviation). For combined treatment, all ratios show significant effects, except for cisplatin and picoplatin; for pretreatment, all ratios show significant effects, except for JMII8 and etoposide. 
set of experiments, DMS153 SCLC cells were pretreated with DCA $10 \mathrm{mM}$ in tissue culture for three days and thereafter tested for their chemosensitivity against the same compounds as described above (Figure 1). With the exception of JM118 and etoposide, all other drugs proved to be less effective, yielding $\mathrm{IC}_{50}$ values that were increased by approximately $100 \%$. Similar results were found for the DCA-preincubation experiments using the NCI-H417 SCLC cell line $(11.0 \% \pm 7.1 \%$ inhibition by DCA alone). Hence, with the exception of oxoplatin (sensitization ratio $1.16 \pm 0.03)$ and etoposide $(0.85 \pm 0.17)$, all other compounds revealed lower toxicity (cisplatin $0.56 \pm 0.22$, carboplatin $0.32 \pm 0.04$, oxaliplatin $0.69 \pm 0.15$, satraplatin $0.84 \pm 0.24, \mathrm{JM} 1180.16 \pm 0.07$, and picoplatin $0.69 \pm 0.27$ ) Therefore, pretreatment of the two SCLC lines with DCA $10 \mathrm{mM}$ reduced the chemosensitivity of the subsequently applied platinum drugs.

Typical dose-response curves for combinations of cisplatin and satraplatin, respectively, with DCA $10 \mathrm{mM}$ are shown in Figure 2 for the ES-2 ovarian clear cell carcinoma cell line. The platinum compounds were applied in twofold dilution steps concurrent with DCA $10 \mathrm{mM}(13.0 \% \pm 0.21 \%$ inhibition as a single agent) for in vitro testing of this cell line with an incubation time of four days. Whereas the cytotoxicity of cisplatin remained unchanged, the activity of satraplatin was significantly increased by almost fourfold.

An overview of the results of chemosensitivity tests using combinations of DCA with cisplatin, carboplatin, satraplatin, and JM118, involving a panel of different cell lines is presented in the Table 1. DCA at a concentration of $10 \mathrm{mM}$ inhibited the growth of most of the cell lines by up to approximately $22 \%$, with minor effects on a colon cancer and two carcinoid cell lines, as well as growth stimulation in TC252 Ewing's sarcoma cells. The cytotoxic activity of cisplatin was elevated in 1/9, carboplatin in 4/9, satraplatin in 8/9, and JM118 in 7/9 of the cell lines, respectively. In contrast to the other three platinum complexes, cisplatin in combination with DCA exhibited lower cytotoxicity against HEK293 normal epithelial kidney cells. H835 carcinoid cells growing as floating spheroids were unique in that they showed increased resistance to cisplatin, satraplatin, and JM118, when combined with DCA. For the cell lines under investigation, the inhibitory effect of DCA on cell proliferation revealed no correlation with sensitization to the chemotherapeutic drugs.

In preliminary experiments, the cytotoxicity of either cisplatin or etoposide for three days, with subsequent exposure to DCA for another four days, was tested using the two

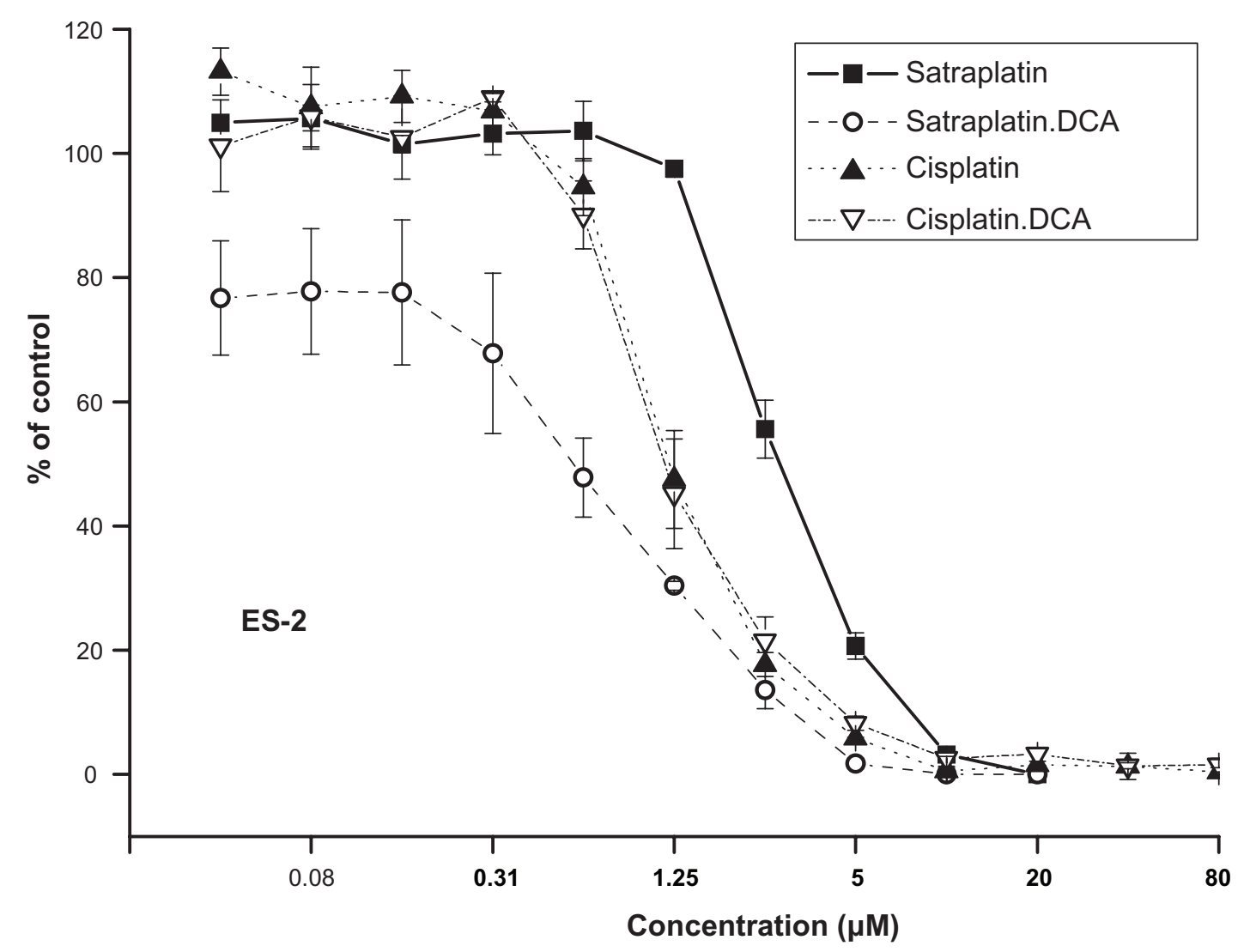

Figure 2 Effects of DCA $10 \mathrm{mM}$ on cytotoxicity of cisplatin and satraplatin against ES-2 ovarian cancer cells (survival in percentage of control, mean \pm standard deviation). 
Table I Effects of combinations of selected platinum drugs with DCA $10 \mathrm{mM}$ on chemosensitivity of a panel of cell lines

\begin{tabular}{|c|c|c|c|c|c|c|c|c|c|c|}
\hline Cell line & Origin & Inhibition & $\begin{array}{l}I_{50} \\
\text { cisplatin }\end{array}$ & $\begin{array}{l}\text { + DCA } \\
\text { ratio }\end{array}$ & $\begin{array}{l}I_{50} \\
\text { carboplatin }\end{array}$ & $\begin{array}{l}+\mathrm{DCA} \\
\text { ratio }\end{array}$ & $\begin{array}{l}I_{50} \\
\text { satraplatin }\end{array}$ & $\begin{array}{l}+D C A \\
\text { ratio }\end{array}$ & $\begin{array}{l}I_{50} \\
\text { JMII }\end{array}$ & $\begin{array}{l}+\mathrm{DCA} \\
\text { ratio }\end{array}$ \\
\hline HEK293 & $\begin{array}{l}\text { Normal } \\
\text { kidney }\end{array}$ & $11.1 \pm 1.8$ & $0.11 \pm 0.07$ & $0.48^{*}$ & $10.0 \pm 3.7$ & $1.47^{*}$ & $1.00 \pm 0.05$ & I.38* & $0.13 \pm 0.07$ & 1.25 \\
\hline DMSI53 & SCLC & $22.5 \pm 6.1$ & $0.04 \pm 0.02$ & 1.12 & $0.37 \pm 0.03$ & $2.4 I^{*}$ & $0.47 \pm 0.06$ & $2.24 *$ & $0.16 \pm 0.07$ & $3.20 *$ \\
\hline $\mathrm{NCl}-417$ & $\mathrm{SCLC}$ & $11.0 \pm 7.1$ & $0.19 \pm 0.06$ & $1.30 *$ & $5.10 \pm 0.07$ & I.80* & $9.8 \pm 0.44$ & $4.73 *$ & $0.87 \pm 0.06$ & $2.80 *$ \\
\hline COLO 205 & $\begin{array}{l}\text { Colon } \\
\text { cancer }\end{array}$ & $3.0 \pm 1.8$ & $11.94 \pm 0.9$ & 0.86 & $54.40 \pm 6.6$ & 1.19 & $16.7 \pm 1.8$ & 1.22 & $1.87 \pm 0.18$ & I.II \\
\hline TC7I & $\begin{array}{l}\text { Ewing's } \\
\text { sarcoma }\end{array}$ & $10.7 \pm 6.8$ & $0.26 \pm 0.04$ & 1.13 & $17.8 \pm 7.7$ & 1.08 & $0.83 \pm 0.08$ & I.80* & $0.09 \pm 0.02$ & $1.27^{*}$ \\
\hline TC252 & $\begin{array}{l}\text { Ewing's } \\
\text { sarcoma }\end{array}$ & $-22.4 \pm 5.2$ & $0.048 \pm 0.008$ & 1.19 & $1.25 \pm 0.07$ & 0.83 & $0.28 \pm 0.07$ & $1.64 *$ & $0.06 \pm 0.01$ & $1.77^{*}$ \\
\hline UMC-II & Carcinoid & $22.2 \pm 3.2$ & $9.90 \pm 0.52$ & 1.22 & $13.30 \pm 2.5$ & $2.49 *$ & $3.75 \pm 0.92$ & $1.50 *$ & $0.91 \pm 0.05$ & I.75* \\
\hline H835 & Carcinoid & $1.0 \pm 1.4$ & $0.63 \pm 0.07$ & 0.53 & $20.0 \pm 6.7$ & 1.12 & $0.86 \pm 0.04$ & $0.5^{*}$ & $0.15 \pm 0.05$ & $0.3 I^{*}$ \\
\hline ES-2 & $\begin{array}{l}\text { Ovarian } \\
\text { cancer }\end{array}$ & $|3.0 \pm 0.2|$ & $0.31 \pm 0.04$ & 1.00 & $6.66 \pm 0.8$ & 1.14 & $2.92 \pm 0.08$ & $2.33^{*}$ & $0.62 \pm 0.02$ & $1.32 *$ \\
\hline
\end{tabular}

Notes: Growth inhibition by DCA $10 \mathrm{mM}$ in percentage of medium control, IC $\mathrm{C}_{50}$ of cisplatin and carboplatin in $\mu \mathrm{g} / \mathrm{mL}$, of satraplatin and JMII 8 in $\mu \mathrm{M}$; + DCA ratio lists $\mathrm{IC}$. in medium control divided by $\mathrm{IC}_{50}$ in presence of DCA $10 \mathrm{mM}$; $* \mathrm{P}<0.05$.

Abbreviations: SCLC, small cell lung cancer; DCA, dichloroacetate.

SCLC lines, DMS153 and NCI-H417, respectively. These regimens resulted in minor sensitization of the cisplatin- and etoposide-pretreated cells to growth inhibition by DCA (data not shown).

\section{Discussion}

DCA is a generic drug that inhibits PDK and, due to its ability to suppress aerobic glycolysis in favor of mitochondrial respiration, has been used medically without approval for the treatment of metabolic acidosis at high doses for decades. ${ }^{16}$ After demonstration of the anticancer activity of DCA in an experimental animal model without apparent side effects, the drug was further investigated in vitro and in vivo using various cancer cell lines. ${ }^{4}$ Meanwhile, DCA is in use as an alternative anticancer drug, and a number of clinical studies have progressed to different stages (registered under clinicaltrials.gov). ${ }^{17}$

The anticancer activity of DCA is attributed to inhibition of PDK and suppression of the pyruvate dehydrogenase complex, which depresses mitochondrial functions that in turn provide crucial factors involved in apoptotic cell death. ${ }^{4}$ Production of lactic acid is thereby diminished, and the mitochondrial membrane potential is normalized concomitant with selective activation of mitochondrial potassium channels in cancer cells. These effects of DCA were reported to be mimicked by silencing of PDK using siRNA. ${ }^{4}$ In vitro treatment of a range of cell lines resulted in variable proportions of cell cycle-arrested or apoptotic cells depending on the tissue of origin in different studies. For example, in colon cancer cell lines, DCA $20 \mathrm{mM}$ cause a selective increase in $\mathrm{G} 2$ phase-blocked and apoptotic cells after two days. ${ }^{6}$ DCA reduced lactate levels in cell culture supernatants and induced dephosphorylation of the E1 $\alpha$ subunit of the pyruvate dehydrogenase complex in all cancer cell lines, leading to decreased proliferation of colorectal cancer but not of noncancerous cells. Recently, DCA was shown to promote the apoptotic machinery through interaction with Bcl-2. ${ }^{18}$ This drug alone produced significant cytotoxic effects in prostate cancer cell lines, was associated with G1 cell-cycle arrest, and apoptotic cells were furthermore demonstrated in DCA-treated endometrial cancer cells. ${ }^{5}$ It should be noted that a recent publication reported conflicting results, identifying higher $\mathrm{IC}_{50}$ values for the anticancer activity of DCA and lack of sensitivity for tumor cells, among others. ${ }^{19}$

Since single use of DCA may be insufficient to achieve significant clinical activity, combination with cytotoxic drugs is expected to potentiate antitumor therapy by converting damage to cellular components into increased apoptotic cell death due to reactivation of mitochondrial function in cancer cells. This approach was first followed in the development of a cisplatin coordination complex containing two axial DCA moieties, namely mitaplatin. However, the cytotoxic activity of mitaplatin only equals that of the parent drug cisplatin, and we found in our laboratory that DCA had synergistic antitumor effects that were restricted to platinum compounds other than cisplatin against a chemoresistant carcinoid cell line. ${ }^{11,15}$ DCA at concentrations $\leq 10 \mathrm{mM}$ inhibited the growth of a large panel of pediatric tumor cell lines only moderately, even though phosphatidylserine was externalized and the 
mitochondrial membrane potential reduced. ${ }^{14}$ Furthermore, DCA abrogated the cytotoxicity of cisplatin in most cell lines without impairment of the in vitro stability or the cellular uptake of cisplatin.

In the present study, we investigated combined treatment of platinum complexes with DCA in a range of different cell lines, including HEK293 normal epithelial kidney cells. Supplementation of DCA in cytotoxicity assays sensitized tumor cells to selected platinum-based drugs, such as carboplatin, satraplatin, and its main metabolite, JM118, in addition to oxoplatin, but not cisplatin, oxaliplatin, and picoplatin. There was no correlation of the effects of DCA with the chemical structure of the compounds or oxidation status of the central platinum atom, respectively, since both the platinum (II) compounds (cisplatin, carboplatin, oxaliplatin, and picoplatin) and the platinum (IV) complexes (satraplatin and oxoplatin) exhibited varying effects with DCA. Given that DCA restores mitochondrial functions and facilitates apoptotic cell death, pretreatment of cancer cells with this drug prior to application of cytotoxic drugs was expected to result in synergism. Instead, preincubation of two SCLC cell lines with DCA $10 \mathrm{mM}$ for three days followed by treatment with platinum drugs yielded antagonistic effects in almost all cases. Similarly, inversion of the treatment schedule, ie, pretreatment with cisplatin or etoposide and subsequent application of DCA, resulted in minor sensitization of the cancer cells to growth inhibition by DCA. Proliferation of one Ewing's sarcoma cell line was stimulated by DCA, and the modulating effects of DCA were low in COLO 205 colon cancer cells and reversed in H835 carcinoid cells, with the exception of cisplatin. H835 spherical aggregates may contain a number of hypoxic cells in their interior that seem to respond in a different manner to DCA. This is in line with a report describing growth stimulation by DCA in HeLa and PANC-1 cancer cell lines under anaerobic conditions. ${ }^{20}$

In conclusion, DCA must be present concurrently to sensitize cancer cells to selected platinum-based drugs, and pretreatment with DCA that is supposed to induce a metabolic switch increases drug resistance to platinum-based drugs, which has to be taken into account with the complementary use of this drug. The mechanisms leading to synergy of DCA with some platinum drugs, especially carboplatin, satraplatin, and JM118, are not clear. High doses of DCA (approximately $50 \mathrm{mg} / \mathrm{kg}$ ) are used clinically to treat metabolic acidosis, and biotransformation of DCA by glutathione $S$-transferase $\zeta$ in the liver is the major elimination pathway in humans. ${ }^{21}$ DCA was shown to suppress the expression of several cytochrome P450 enzymes and glutathione S-transferase $\pi 1$, by two- to fivefold in rodents. ${ }^{9}$ Therefore, DCA may downregulate drug-metabolizing enzymes that inactivate platinum compounds. DCA is generally well tolerated and dehalogenated to monochloroacetate and glyoxylate in vivo before it is further catabolized to glycolate, glycine, oxalate, and carbon dioxide. ${ }^{22}$ Because DCA may be present in quite high intracellular concentrations, these products of drug breakdown may directly modulate the intracellular fate of different platinum drugs. DCA may represent a valuable adjunct to third-generation platinum drugs for the treatment of cisplatin-refractory cancer entities, such as SCLC, Ewing's sarcoma, and ovarian cancer. ${ }^{23}$

\section{Acknowledgment}

This study was supported by a fund from the Jubilaeumsfonds (Oesterreichische Nationalbank, Grant Number 13345).

\section{Disclosure}

The authors report no conflicts of interest in this work.

\section{References}

1. Gogvadze V, Zhivotovsky B, Orrenius S. The Warburg effect and mitochondrial stability in cancer cells. Mol Aspects Med. 2010;31:60-74.

2. van der Heiden MG, Cantley LC, Thompson CB. Understanding the Warburg effect: The metabolic requirements of cell proliferation. Science. 2009;324:1029-1033.

3. Michelakis ED, Webster L, Mackey JR. Dichloroacetate (DCA) as a potential metabolic-targeting therapy for cancer. Br J Cancer. 2008;99: 989-994.

4. Bonnet S, Archer SL, Allalunis-Turner J, et al. A mitochondria- $\mathrm{K}^{+}$ channel axis is suppressed in cancer and its normalization promotes apoptosis and inhibits cancer growth. Cancer Cell. 2007;11:37-51.

5. Wong JY, Huggins GS, Debidda M, et al. Dichloroacetate induces apoptosis in endometrial cancer cells. Gynecol Oncol. 2008;109:394-402.

6. Madhok BM, Yeluri S, Perry SL, et al. Dichloroacetate induces apoptosis and cell-cycle arrest in colorectal cancer cells. Br J Cancer. 2010;102:1746-1752.

7. Sun RC, Fadia M, Dahlstrom JE, et al. Reversal of the glycolytic phenotype by dichloroacetate inhibits metastatic breast cancer cell growth in vitro and in vivo. Breast Cancer Res Treat. 2010;120:253-260.

8. Kaufmann P, Engelstad K, Wei Y, et al. Dichloroacetate causes toxic neuropathy in MELAS: A randomized, controlled clinical trial. Neurology. 2006;66:324-330.

9. Thai SF, Allen JW, deAngelo AB, et al. Altered gene expression in mouse livers after dichloroacetic acid exposure. Mutat Res. 2003;543: 167-180.

10. Sedletska Y, Giraud-Panis MJ, Malinge JM. Cisplatin is a DNAdamaging antitumour compound triggering multifactorial biochemical responses in cancer cells: Importance of apoptotic pathways. Curr Med Chem Anticancer Agents. 2005;5:251-265.

11. Dhar S, Lippard SJ. Mitaplatin, a potent fusion of cisplatin and the orphan drug dichloroacetate. Proc Natl Acad Sci U S A. 2009;106: 22199-22204

12. Tegeder I, Bräutigam L, Seegel M, et al. Cisplatin tumor concentrations after intraarterial cisplatin infusion or embolization in patients with oral cancer. Clin Pharmacol Ther. 2003;73:417-426.

13. Bowker-Kinley MM, Davis WI, Wu P, et al. Evidence for existence of tissue-specific regulation of the mammalian pyruvate dehydrogenase complex. Biochem J. 1998;329:191-196. 
14. Heshe D, Hoogestraat S, Brauckmann C, et al. Dichloroacetate metabolically targeted therapy defeats cytotoxicity of standard anticancer drugs. Cancer Chemother Pharmacol. 2010 May 26. [Epub ahead of print].

15. Fiebiger W, Olszewski U, Ulsperger E, et al. In vitro cytotoxicity of novel platinum-based drugs and dichloroacetate against lung carcinoid cell lines. Clin Translat Oncol. 2010. In press.

16. Fields AL, Wolman SL, Halperin ML. Chronic lactic acidosis in a patient with cancer: Therapy and metabolic consequences. Cancer. 1981;47:2026-2029.

17. Pearson H. Cancer patients opt for unapproved drug. Nature. 2007;446: 474-475

18. Cao W, Yacoub S, Shiverick KT, et al. Dichloroacetate (DCA) sensitizes both wild-type and over expressing Bcl-2 prostate cancer cells in vitro to radiation. Prostate. 2008;68:1223-1231.
19. Stockwin LH, Yu SX, Borgel S, et al. Sodium dichloroacetate (DCA) selectively targets cells with defects in the mitochondrial ETC. Int J Cancer. 2010 Jun 7. [Epub ahead of print].

20. Anderson KM, Jajeh J, Guinan P, et al. In vitro effects of dichloroacetate and CO2 on hypoxic HeLa cells. Anticancer Res. 2009;29. $4579-4588$.

21. Li T, Schultz I, Keys DA, et al. Quantitative evaluation of dichloroacetic acid kinetics in human - a physiologically based pharmacokinetic modeling investigation. Toxicology. 2008;245:35-48.

22. Stacpoole PW, Henderson GN, Yan Z, et al. Clinical pharmacology and toxicology of dichloroacetate. Environ Health Perspect. 1998; 106:989-994.

23. Olszewski U, Hamilton G. A better platinum-based anticancer drug yet to come? Anticancer Agents Med Chem. 2010;10:293-301.

\section{Publish your work in this journal}

Clinical Pharmacology: Advances and Applications is an international, peer-reviewed, open access journal publishing original research, reports, reviews and commentaries on all areas of drug experience in humans. The manuscript management system is completely online and includes a very quick and fair peer-review system, which is all easy to use
Visit http://www.dovepress.com/testimonials.php to read real quotes from published authors. 